MODELING, IDENTIFICATION AND CONTROL, 1992, vOL. 13, NO. 1, 41-49

doi:10.4173/mic.199214

\title{
A method for the control of alumina concentration in aluminium reduction cells
}

\author{
JENS G. BALCHEN $\dagger$
}

Keywords: Experimenting control, aluminium reduction cell, alumina concentration.

\begin{abstract}
The paper presents a new method for the control of the concentration of the alumina in electrolysis cells for the production of aluminium. The method is based upon the well known fact that apparent resistivity of the cell is a function of the alumina concentration so that the resistivity has the lowest value around the concentration of three per cent and increases in both directions. The method uses the cross correlation between a perturbation of the feed flow of alumina into the cell and the resulting response in measured voltage across the cell. The cross-correlation is proportional to the slope of the resistivity against concentration curve, making it possible to control the alumina flow, to achieve a desired slope. The method has much in common with other methods presently in use which require a much more complicated computation scheme.
\end{abstract}

\section{Aluminium electrolysis cell}

Figure 1 shows a sketch of a modern aluminium electrolysis cell. It consists of a number of prebaked carbon anodes hanging down from large horizontal aluminium metal beams conducting the electrolysis DC current (about 150000-250000 A). The carbon anodes are partly submerged in the electrolyte which consists mainly of molten cryolite $\left(3(\mathrm{NaF}) \mathrm{AlF}_{3}\right)$ and aluminium oxide $\left(\mathrm{Al}_{2} \mathrm{O}_{3}\right)$. There are also other chemical agents to control the electrolyte properties. The temperature of the electrolyte is around $950-970^{\circ} \mathrm{C}$. When DC current passes through the electrolyte from the anodes, aluminium metal is produced which is slightly heavier than the electrolyte, this sinks to

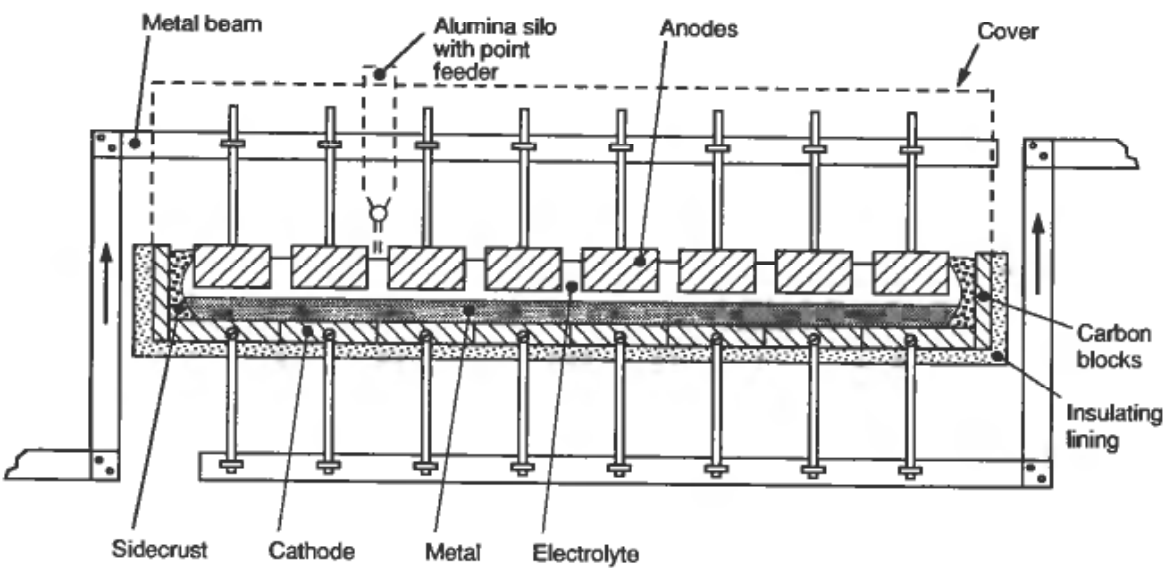

Figure 1. Simplified cross-section of aluminium reduction cell with prebaked anodes.

Received 11 March 1992.

$\dagger$ Division of Engineering Cybernetics, The Norwegian Institute of Technology, 7034 Trondheim, Norway. 
form a cathode which floats on a carbon lining surrounded by insulating material located in a heavy steel case.

The main reaction of the aluminium electrolysis is

$$
2 \mathrm{Al}_{2} \mathrm{O}_{3}+3 \mathrm{C} \rightarrow 4 \mathrm{Al}+3 \mathrm{CO}_{2}
$$

in which the carbon of the anode is active in the reduction process. Because of the very small interpolar distance between the anodes and the metal cathode (about $5 \mathrm{~cm}$ ), there is a reverse process going on according to the equation

$$
2 \mathrm{Al}+3 \mathrm{CO}_{2} \rightarrow \mathrm{Al}_{2} \mathrm{O}_{3}+3 \mathrm{CO}
$$

This reoxidation is the cause of a loss of efficiency relative to what could have maximally been achieved according to the Faraday number. Modern aluminium reduction cells have a current efficiency $(\eta)$ around $90-93 \%$. This explanation is a simplification of the actual reaction mechanisms.

The total voltage across one cell is about $5 \mathrm{~V}$ of which about $1.2 \mathrm{~V}$ represent the electrochemical potential and the rest is duc to resistivity in the different parts of the electric circuit, the electrolyte is of particular interest here. About 150 such cells are connected in series and are fed from a high capacity rectifier station with precise current control.

Each cell is fed alumina from a silo above the cell at a controllable rate. In modern cells this is done almost continuously whereas in older cells the feeding is discontinuous and imprecise. About $2 \mathrm{~kg}$ of alumina are needed to produce $1 \mathrm{~kg}$ of aluminium. The aluminium produced by the electrolysis and forming the cathode in the bottom of the cell is pumped up into a special transportation tank at regular intervals.

Two physical balances are of particular importance in the operation of an aluminium reduction cell. These are

The alumina balance resulting from the difference between the alumina fed to the cell and the alumina consumed by electrolysis and leading to changes in the alumina concentration in the electrolyte

The energy balance resulting from the difference between electric energy supplied to the cell, the losses to the environment and energy consumed for heating and dissolving alumina etc. Changes in energy balance lead to changes in electrolyte temperature.

The control of an aluminium reduction cell is concerned with these two balances.

Molten cryolite is corrosive and the only material which can withstand it is frozen cryolite. Therefore the cell is so designed that a crust of frozen cryolite will form around its vertical outer walls. When properly designed and operated, this crust will have a well defined thickness and plays an important role in the material balance of the electrolyte. If the temperature is increased for some reason, this crust will tend to melt and vice versa when the temperature is lowered.

The energy balance is controlled by changing the position of the anodes in vertical direction leading to changing interpolar distance. This is achieved by motors lifting and lowering the metal beams above the cell.

\section{Control of alumina concentration}

The earliest developments of modern control systems for aluminium reduction cells were reported in the end of the 1960s (McMahon and Dirth 1966, Balchen and Høsøien 1971, Larsen 1971). Large scale industrial tests of model based control systems for 


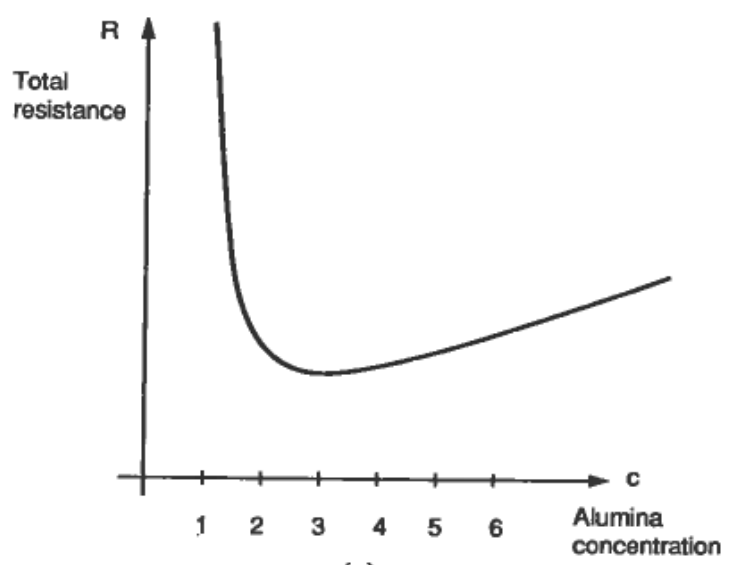

(a)

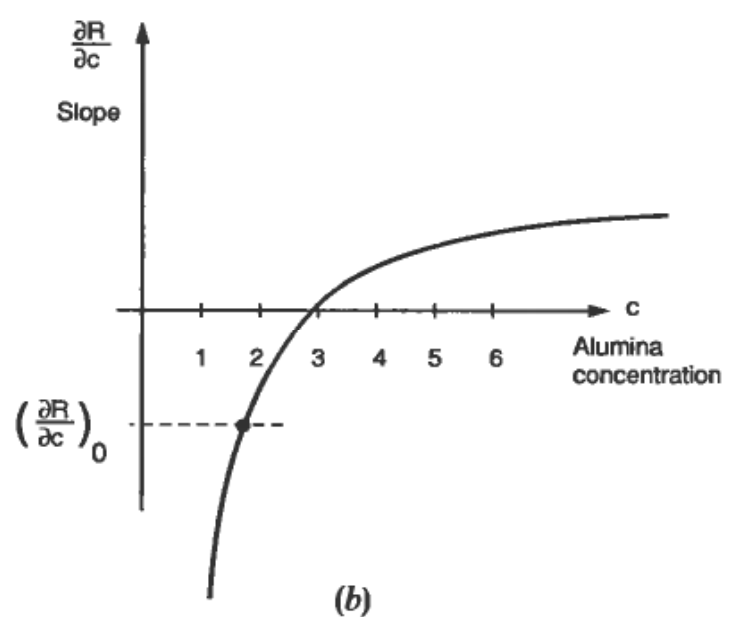

(b)

Figure 2. (a) The total resistance as a function of alumina concentration and $(b)$ the slope of the curve.

reduction cells have been reported (Gran 1980) and a successful implementation of a system performing nearly the same task as that presented in this paper, was reported by Borg et al. (1986).

It is established that the sum of the apparant resistivity of the electrolyte and of the junction between the anodes and the electrolyte is a well-defined function of the alumina concentration in the electrolyte as indicated in Fig. 2(a). The increase in the resistivity at low concentrations is caused mainly by a phenomenon on the junction between anode and electrolyte which can be interpreted as a reduction in conducting area of the anodes or a gradual appearance of nonconducting subareas. At concentrations less than about $1 \%$ the voltage across the cell will rise to above $30 \mathrm{~V}$ producing what is commonly referred to as the anode effect. The curve of Fig. $2(a)$ has its minimum around an alumina concentration of about $3 \%$. At higher concentrations there is again a gradual increase in resistivity caused by the oxide which is in itself an insulator. Depending on temperature the electrolyte may dissolve up to 6-8\% alumina with an increasing tendency of undissolved alumina to collect at the bottom of the cell. Since alumina is heavier than aluminium metal, such undissolved alumina will form an insulating barrier between the carbon and the metal. This is a undesirable condition which must be avoided. One way of doing this is to run the cell at an alumina 
concentration less than $3 \%$, that is where the slope of the curve in Fig. $2(a)$ is fairly steep and negative.

Attempts at operating the cell at a certain level of the resistance itself has proven unsuccessful because the same resistance is occurring at two different alumina concentrations yielding opposite signs of the control loop gains. A more desirable way to control the alumina concentration is thus to control the slope of the resistance curve as indicated in Fig. 2(b).

An easy way to determine the slope of the resistance curve is to find the crosscorrelation between a calculated perturbation of the alumina concentration and the resulting response in the cell voltage. The cross-correlation will be proportional to the slope. This principle of control has been known for more than 40 years (Draper and $\mathrm{Li}$ 1951).

Feeding alumina to the cell from silos above the cell is achieved by one or more motor driven apportioning devices whose speed can be adjusted (about $1-2 \mathrm{~kg} / \mathrm{min}$ ). Figure 3 shows a schematic diagram of a control system for the alumina feeder based upon the slope of the resistance curve determined by cross correlation.

The consumption of alumina due to electrolysis $\left(w_{1}\right)$ which is determined by the current efficiency $(\eta)$ and the cell current $(I)$ is subtracted from the flow of alumina supplied from the feeder $\left(w_{F}\right)$ modified by some mixing dynamics (transfer function $\left.\approx \exp (-\tau s) / 1+T_{c} s\right)$. The difference between these two flows is accumulated in the electrolyte (transfer function $\approx 1 / M s$, electrolyte mass $=M$ ) leading to changes in the alumina concentration $(c)$. The alumina concentration (together with many other variables in the process) results in the total cell resistance $(R)$ which, when multiplied by the cell current $(I)$ yields the total cell voltage $(U)$.

The motor driven feeder supplying the alumina feed $\left(w_{F}\right)$ has a velocity $(n)$ which is the sum of an average velocity $(\bar{n})$ supplied by the controller and a perturbation velocity $(\Delta n)$ supplied by an oscillator. The perturbation may either have the form of a sine wave, (magnitude $\Delta n$, frequency $\omega_{0}$ ) or a square wave. Theoretically the best wave form is a sine wave, but in practice a square wave may be simpler to implement and therefore more desirable.

The total cell resistance

$$
R=R_{0}+R_{a, b}+R_{b}
$$

can be regarded as the sum of three elements, $R_{0}$ (connectors, anode, aluminium, cathode), $\boldsymbol{R}_{a, b}$ (the anode to electrolyte bath junction) and $\boldsymbol{R}_{b}$ (electrolyte).

$R_{0}$ may be regarded as reasonably constant. A simplified formula for $R_{a, b}$ is

$$
R_{a, b} \cong \frac{k_{1}}{c-c_{x}}
$$

where $k_{1}$ is a constant depending on temperature etc. and $c_{x}$ is the critical alumina concentration determining the anode effect.

The bath resistance can be described approximately by

$$
R_{b} \approx k_{2} d c
$$

where $k_{2}$ is a constant depending on temperature etc. and $d$ is the interpolar distance.

Equations (3), (4) and (5) may be used to estimate approximately the voltage change that will occur due to changes in interpolar distance $(d)$ when the anode is adjusted to correct for its consumption or during the removal of aluminium metal. Since the cell current is relatively constant and if the control system of Fig. 3 is blocked during anode adjustments, then the compensation just mentioned can be neglected. 


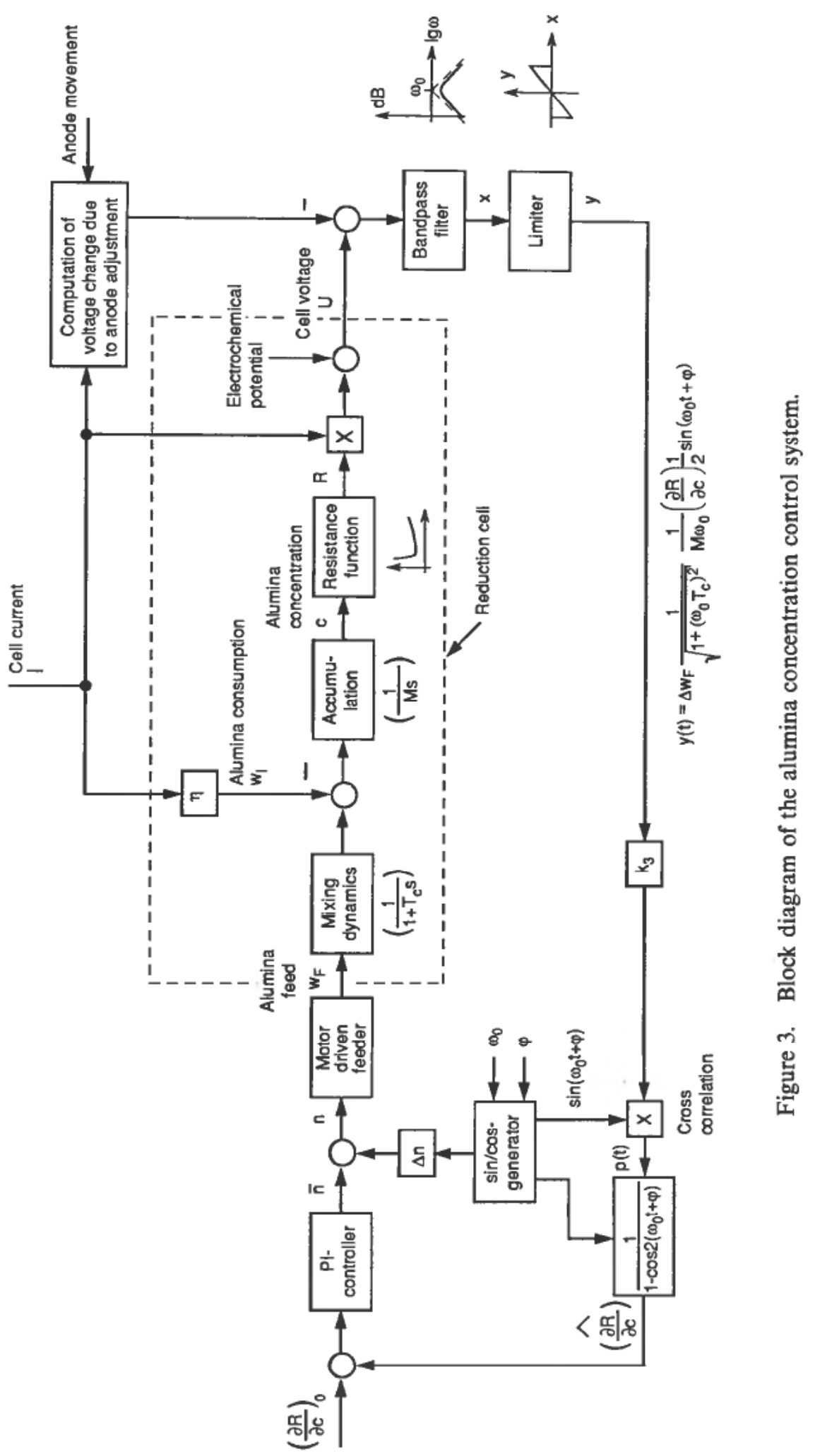


The measured cell voltage is fed to a bandpass filter. In a continuous implementation this should have a transfer function

$$
h_{f}(s)=\frac{s / \omega_{0}}{\left(1+s / \omega_{0}\right)^{2}}
$$

In a discrete implementation the transfer function will become

$$
h_{f}\left(z^{-1}\right)=\frac{\omega_{0} T / 2}{\left(\omega_{0} T / 2-1\right)^{2}} \cdot \frac{\left(1-z^{-2}\right)}{\left(\frac{\omega_{0} T / 2+1}{\omega_{0} T / 2-1}+z^{-1}\right)^{2}}
$$

in which $z^{-1}$ is the discrete delay operator and $T$ is the sampling period.

The purpose of the band pass filter is to remove all constant and slowly varying components in the cell voltage and to filter out high frequency noise. By making the band pass centre frequency equal to the oscillation frequency $\left(\omega_{0}\right)$ the phase shift of the perturbation signal in the band pass filter will equal zero.

Following the band pass filter is a limiter whose nonlinear characteristic is indicated in Fig. 3. Its purpose is to remove all signals which are caused by transients in the system and are larger than the normal perturbations in the cell voltage due to the perturbations in the alumina feed.

The resulting voltage output of the limiter will become

$$
y(t)=\Delta w_{F} \frac{1}{\left(1+\left(\omega_{0} T_{c}\right)^{2}\right)^{1 / 2}} \frac{1}{M \omega_{0}}\left(\frac{\partial R}{\partial c}\right) \frac{1}{2} \sin \left(\omega_{0} t+\phi\right)
$$

The phase shift $\phi$ is going to be more than $\left(-90^{\circ}\right)$ due to the mixing dynamics and the accumulation (integration). It is seen from (9) that the signal is proportional to the slope of the resistance function $(\partial R / \partial c)$. The signal $y(t)$ is multiplied by a factor

$$
k_{3}=\frac{4}{\Delta w_{F}}\left(1+\left(\omega_{0} T_{c}\right)^{2}\right)^{1 / 2} M \omega_{0}
$$

and the result is crosscorrelated (multiplied) with the phase shifted perturbation signal $\left(\sin \left(\omega_{0} t+\phi\right)\right)$ resulting in the signal

$$
p(t)=\frac{\partial R}{\partial c}\left(1-\cos 2\left(\omega_{0} t+\phi\right)\right)
$$

In other words we have made an estimate of the slope of the resistance curve, but this estimate has a oscillating corrective term with the frequency $2 \omega_{0}$. This term can be removed by division as indicated in Fig. 3 since the input oscillation is known. This particular operation should be performed with caution since the corrective term assumes the value zero every period and leaves a singular situation. One way of avoiding this problem is to block the division when the denominator is less than a certain numerical value.

When the estimate of the slope of the resistance function $(\partial R / \partial c)$ has been determined, it is subtracted from the desired slope $(\partial R / \partial c)_{0}$ and the deviation is fed to a PI-controller whose output is the average value of the speed $(\bar{n})$ of the motor driven feeder. The proportional action of this controller is necessary for stability reasons because the control loop contains two integrating elements.

The phase shift $(\phi)$ of the crosscorrelation signal indicated in Fig. 3 must be adjusted so as to compensate for the equivalent $\operatorname{lag}\left(\phi_{c}\right)$ in the process. This compensation is not very critical. The amplitude of perturbation must be $\Delta n<\bar{n}$. 
The frequency of perturbation $\left(\omega_{0}\right)$ should be chosen as high as possible, but low enough to give an acceptable response in the alumina concentration relative to the natural fluctuations (noise). This will be achieved when $\omega_{0} \cong 1 / T_{c}$ and $\Delta n \approx 0.5 \bar{n}$. A reasonable estimate of $T_{c}$ is $T_{c} \approx 5-20(\mathrm{~min})$ leading to $\omega_{0} \approx 0.05-0.2(\mathrm{rad} / \mathrm{min})$ equivalent to a perturbation period $T_{0} \approx 30-120(\mathrm{~min})$. The settling time of the control system after a step disturbance may be expected to be about $4 T_{0}-10 T_{0}$.

\section{Simulation of system performance}

A number of simulation studies have been performed by students at the Division of Engineering Cybernetics, The Norwegian Institute of Technology. One such study is reported in Fjellstad and Hagen (1989)) and is the basis for the following. The cell is assumed to have a total mass of electrolyte $M=5000 \mathrm{~kg}$, a nominal cell current $I=250 \mathrm{kA}$, a current efficiency $\eta=0.90$, which leads to a nominal consumption of alumina $\bar{w}=2.69 \mathrm{~kg} / \mathrm{min}=0.045 \mathrm{~kg} / \mathrm{s}$. One $\mathrm{kg}$ of alumina will change the alumina concentration in the electrolyte by $0.02 \%$ in this study it is proposed to perturb the alumina concentration by an amount $\Delta c=0.1 \%$ around a nominal desired value of $\bar{c}=1.8 \%$. If the perturbation in alumina flow is assumed to $100 \%$, that is $\Delta w=\bar{w}$, the shortest possible perturbation period can be computed to be $T_{0}=2 \pi / \omega_{0}=700$ (s). This period is probably around $50 \%$ of what will be a practical value, but is chosen in this study.

Figure $4(a)$ shows the flow of alumina, Fig. 4(b) the oxide concentration, Fig. 4(c) the variations in the cell resistance due to oxide perturbation and Fig. $4(d)$ the resulting estimate of the gradient $(\partial \hat{R} / \partial c)$. In the experiment the initial oxide

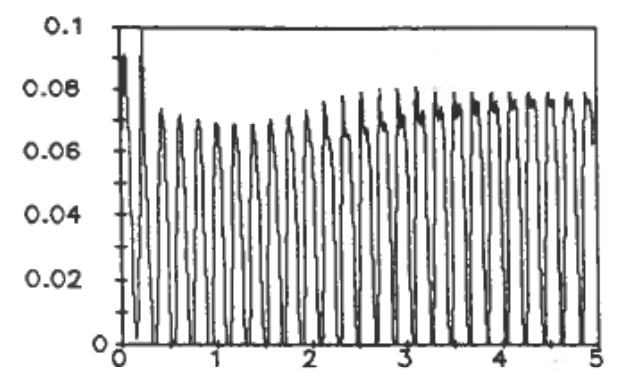

(a)

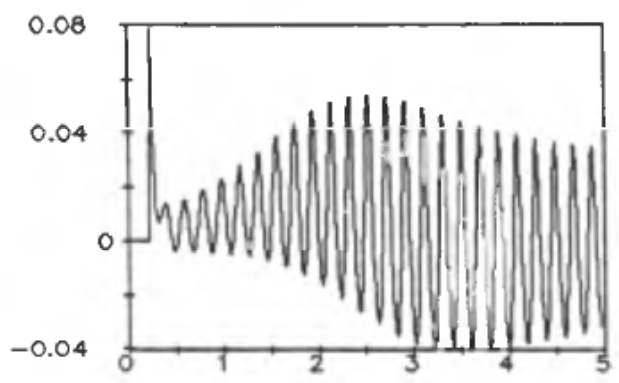

(c)

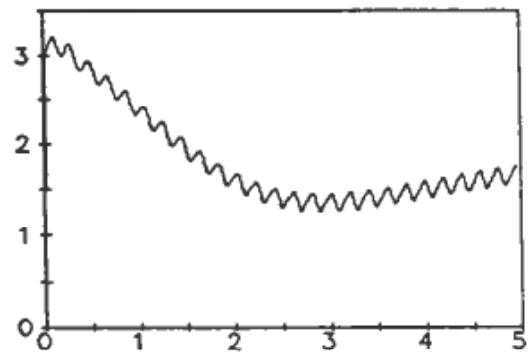

(b)

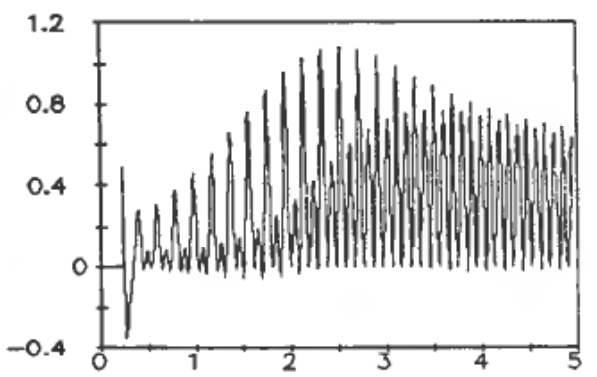

(d)

Figure 4. (a) Alumina flow ( $\left.w_{F}\right)$. (b) Alumina concentration (c). (c) Resistance variation. (d) Estimated gradient $(\partial \hat{R} / \partial c)$. 


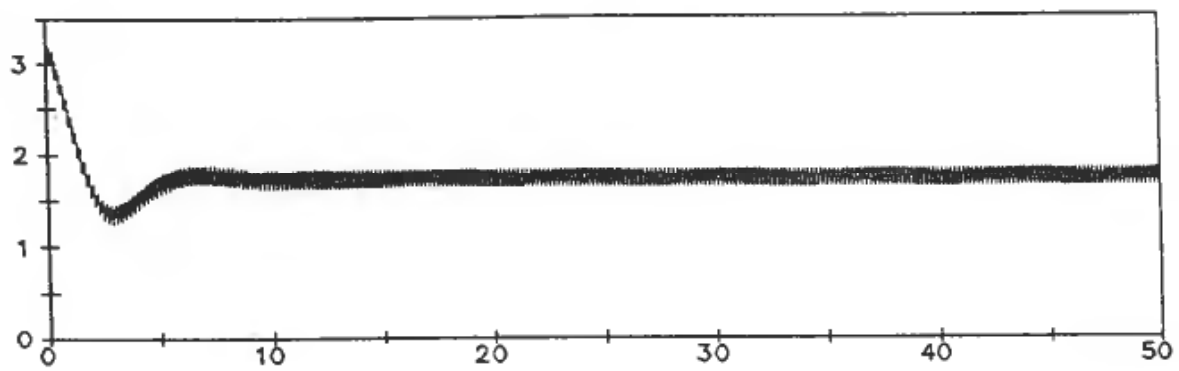

Figure 5. Alumina concentration (c).

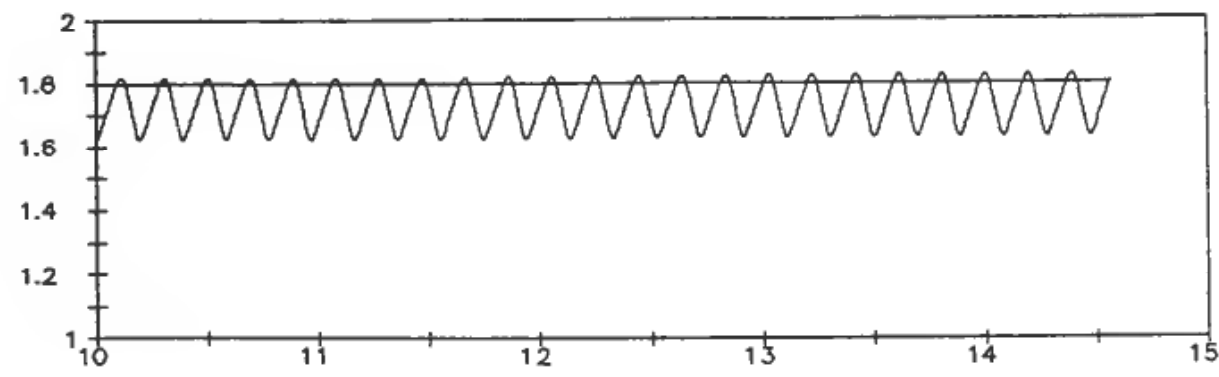

Figure 6. Steady state error in concentration due to nonlinearity.

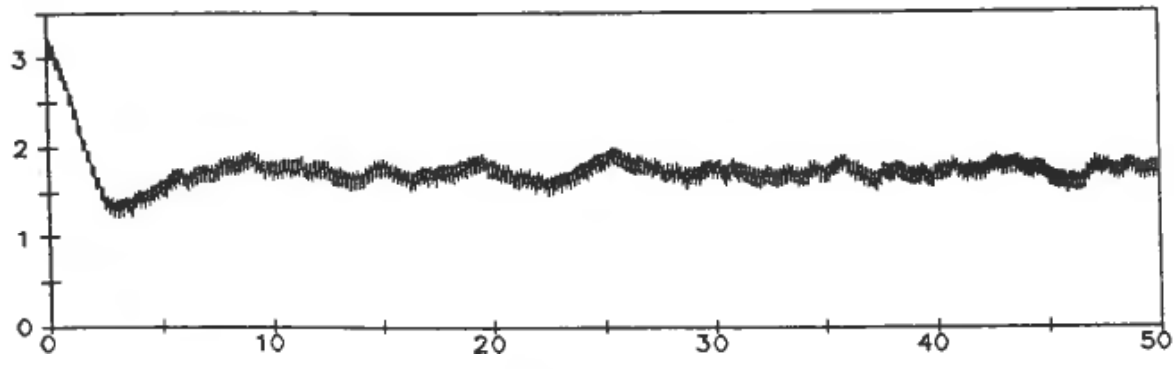

Figure 7. Alumina concentration $(c)$ when process is disturbed.

concentration is assumed to be $c_{0}=3(\%)$. The PI-controller has integral time $T_{i}$ $=3500(\mathrm{~s})$ and proportional gain $K_{p}=0-05$. Figure 5 shows the same curve as in Fig. $4(b)$, but with a different time scale. As can be seen, the settling time is around $4 \mathrm{~h}$ for this particular tuning of the system parameters. Figure 6 shows the consequence of the fact that the resistance of the cell is a nonlinear function of the alumina concentration. The resistance perturbations will not be ideally sinusoidal leading to a slight steady-state error in the concentration relative to the desired value. As is seen, this error is less than about $0.1 \%$.

The previous simulations were done with an ideal process model without any noise. If a process disturbance with the character of white noise with a standard deviation of $0.033(\% / \mathrm{s})$ is assumed in the flow of alumina and the voltage measurement across the cell is assumed to have a additional white noise with standard deviation of $0.01(\%)$, the control of the oxide concentration will perform as is shown in Fig. 7. This result should be compared with that shown in Fig. 5 and is quite satisfactory. 


\section{Conclusions}

The very simple control system discussed above, has a number of advantages compared to those reported in the literature. The most important advantages are:

Extreme simplicity leading to low demand on computer capacity and computer time.

Short settling time after disturbance.

Robustness to large variations in oxide concentration.

\section{References}

BALCHEN, J. G., and Høsøren, O. (1971). Investigation of different strategies for computer control of aluminium reduction cells. 3rd IF AC/IFIP Conference of Digital Computer Applications to Progress Control, Helsinki. Instrument Society of America, 1971, Part I, paper III-5, pp, 1-8.

BORG, P., Moen, T. and AalBU, J. (1986). Adaptive control of alumina reduction cells with point feeders. Modeling, Identification and Control, 7, 45-56.

DraPer, C. S. and LI, Y. T. (1951), Principles of optimalizing control and an application to internal combustion engine. American Society of Mechanical Engineering Publication.

FJelLSTAD, O.-E. and HAGEN, J. (1989). Experimenting control of alumina concentration in aluminum electrolysis cells (in Norwegian). Project Report. Div. of Eng. Cybernetics, The Norwegian Institute of Technology, Trondheim, Norway.

Gran, E. (1980). Multivariable control of aluminium reduction cells. Modeling, Identification and Control, 1, 247-258.

LARSEN, A. S. (1971). The integrated on-line commercial computer installation at the Aluminium reduction plant in Sunndal, Norway. The 3rd IF AC/IFIP Conference on Digital Computer Applications to Process Control, Helsinki. Instrument Society of America, 1971, Part I, paper III-6, pp. 1-7.

MCMAHON. T. K. and DirTh, G. P. (1966) Computer control of aluminum reduction cells. Journal of Metals, 18, 317-319. 